\title{
Utility of endoscopic retrograde cholangiopancreatography in infant patients with conservational endoscopy
}

\author{
Mingzhe Weng ${ }^{1 \#}$, Lubing Wang ${ }^{2 \#}$, Hao Weng ${ }^{1}$, Jun Gu ${ }^{1}$, Xuefeng Wang ${ }^{1}$ \\ ${ }^{1}$ Department of General Surgery, Xinhua Hospital Affiliated to Shanghai Jiao Tong University School of Medicine, Shanghai, China; ${ }^{2}$ Department of \\ General Surgery, Xinhua Hospital Affiliated to Shanghai Jiao Tong University School of Medicine, Chongming Branch, Shanghai, China \\ Contributions: (I) Conception and design: X Wang; (II) Administrative support: X Wang, J Gu; (III) Provision of study materials or patients: M Weng, \\ L Wang; (IV) Collection and assembly of data: M Weng, H Weng; (V) Data analysis and interpretation: M Weng; (VI) Manuscript writing: All \\ authors; (VII) Final approval of manuscript: All authors. \\ "These authors contributed equally to this work. \\ Correspondence to: Xuefeng Wang, MD. Division Chief, Department of General Surgery, Xinhua Hospital Affiliated to Shanghai Jiao Tong University \\ School of Medicine, Shanghai 200092, China. Email: wxxfd@live.cn.
}

\begin{abstract}
Background: The indications for endoscopic retrograde cholangiopancreatography (ERCP) in infant patients (age $<1$ year) differ from those in adults. A paucity of data and concerns about the potential lower effectiveness and more adverse effects limit its utility, even in tertiary care centres. In this study, we retrospectively analysed the indications, success rates, and adverse effects of ERCP in these groups.

Methods: From June 2014 to March 2018, 17 ERCPs were performed in 15 children [median age: 10.4 months (6-12 months); median weight: $6.6 \mathrm{~kg}(3.3-10.7 \mathrm{~kg})]$. A conventional duodenoscope was utilized in all procedures. All patients were followed up as inpatients.

Results: Fifteen therapeutic and two diagnostic procedures were managed by licensed paediatric endoscopist. Successful cannulation was obtained in all patients (100\%). A high proportion (47.1\%) of pancreaticobiliary maljunction (PBM) and therapeutic procedures $(88.2 \%)$ were identified. Two cases of mild pancreatitis were recorded, accounting for $12.5 \%$ of the post-ERCP pancreatitis (PEP) rate. By multivariable analysis, recurrent acute pancreatitis and pancreatic duct (PD) cannulation/injection were identified as PEPrelated risk factors. All complications were managed conservatively.

Conclusions: ERCP in infant patients $(0.5<$ age $<1$ year) could be safely completed by conservational endoscopy. A high proportion of PBM and therapeutic procedures were identified in our study cohort. The overall adverse-event rate was acceptable, and no serious complication occurred. The PEP-related independent risk factors included recurrent acute pancreatitis and PD cannulation/injection.
\end{abstract}

Keywords: Endoscopic retrograde cholangiopancreatography (ERCP); neonatal and infant patients; conventional endoscopy; pancreaticobiliary maljunction; post-ERCP pancreatitis

Submitted Aug 05, 2021. Accepted for publication Oct 14, 2021.

doi: 10.21037/tp-21-406

View this article at: https://dx.doi.org/10.21037/tp-21-406

\section{Introduction}

Endoscopic retrograde cholangiopancreatography (ERCP) has been widely applied for the diagnosis and treatment of biliary and pancreatic disorders in adults, but is rarely used in paediatric patients. The subcommittee on Endoscopy and Procedures of the Patient Care Committee of the
North American Society for Paediatric Gastroenterology systematically summarized the utility of ERCP in children (1), and many paediatric experts have engaged in this topic (2-9); however, consensus about effectiveness or indications of ERCP in children has not been achieved, let alone infant patients (age $<1$ year). Infant patients differ considerably from older children in terms of anatomic and 
physiological aspects. Very few studies have exclusively involved infant patients (10). The success rates from stratified data vary considerably, from $27 \%$ to $95 \%$ (11).

The limited utility of ERCP in infants results from concerns about its effectiveness and safety. ERCP is an invasive procedure with a post-ERCP pancreatitis (PEP) rate of $5-7 \%$ in adult cohorts (12), which was reported to increase in paediatric patients (13). Moreover, the qualifications of the endoscopist are a contentious topic. Owing to the rarity of cases and lengthy learning curves, paediatric ERCPs are usually performed by adult gastroenterologists who are less familiar with paediatric diseases. Importantly, several studies and guidelines suggest that the minimum age for the use of conventional duodenoscopy may be 1 year $(14,15)$. The paediatric sideviewing duodenoscope with a working channel of $2 \mathrm{~mm}$ (14) can only perform diagnostic procedures that further temper enthusiasm for infant ERCP.

In this paper, we retrospectively analysed infant (age $<1$ year) ERCP performed in our high-volume endoscopy centre to explore the use of this therapeutic procedure with conventional equipment. We present the following article in accordance with the STROBE reporting checklist (available at https://dx.doi.org/10.21037/tp-21-406).

\section{Methods}

Our centre is the only tertiary care paediatric facility in this region, and the paediatric unit provides an in- and outpatient service for children of all ages diagnosed with gastrointestinal, pancreatic, or liver diseases. Diagnosis and treatment for these children is organized by the multidisciplinary team, which includes endoscopists, paediatricians, and paediatric anesthetists. Highend ultrasonography and/or magnetic resonance cholangiopancreatography was available for all patients before ERCP. The ERCP procedures were all performed under general anaesthesia by a licensed paediatric endoscopist (Wang X, MD). All procedures performed in this study involving human participants were in accordance with the Declaration of Helsinki (as revised in 2013). The study was approved by ethics committee of the Xinhua Hospital affiliated to Shanghai Jiao Tong University School of Medicine (ID: 201800051). Individual consent for this retrospective analysis was waived.

A JF260V conventional duodenoscope (Olympus, Tokyo, Japan) was used. Cannulation and sphincterotomy were performed with a papillotome marked Dreamtome
RX44 (Boston Scientific, Natick, USA). Other therapeutic instruments included a Zimmon pancreatic stent (Cook, Bloomington, USA), a nasal biliary drainage catheter (Cook), high-performance guidewires (Boston Scientific, Natick, USA), and a wire-guided balloon dilatation catheter (Boston Scientific, Natick, USA). Carbon dioxide $\left(\mathrm{CO}_{2}\right)$ was used for insufflation to minimize the adverse effects of bowel air distention. The infant patients were placed in the left lateral position, and their eyes, thyroid glands, and sexual glands were specially protected (Figure 1). Diluted contrast medium (Omnipaque) was injected to opacify the entire pancreaticobiliary duct.

Successful intervention was defined as the catheter being cannulated into the common channel and the contrast agent filling at least part of the pancreaticobiliary system. All infants were followed up as inpatients for at least 3 days to allow for strict monitoring. Amylase was evaluated 3 hours, 1 day, and 2 days post-ERCP. PEP was graded according to the 2010 American Society for Gastrointestinal Endoscopy lexicon for adverse events (mild, moderate, severe, or fatal) (13). No PEP prophylactic agent was used, but once $\mathrm{PEP}$ was diagnosed, alleviating protease inhibitors was recommended.

\section{Statistical analysis}

Statistical analysis was managed with SPSS (IBM SPSS Statistics, Version 19). The $\chi^{2}$ test or Fisher's exact test was used for categorical variables evaluating by sample size, and the Student's $t$-test was used for continuous variables. Differences were considered significant at $\mathrm{P}<0.05$ (twosided). Variables with $\mathrm{P}<0.1$ in the univariable analyses were included as candidate predictors. The forward stepwise method was utilized to construct a multiple logistic regression model of the development of PEP. The preliminary univariate tests were taken as exploratory, not requiring correction, as the variables tested were considered in the multivariable logistic regression analysis, which was taken as the main definitive result.

\section{Results}

\section{Interventions}

Infant patients ( $\leq 1$ year old) accounted for approximately $0.1 \%$ of our total ERCP caseload. A typical setting is shown in Figure 1A. From June 2014 to March 2018, 17 ERCPs were performed in 15 children (female/male: 8/7). Patients 

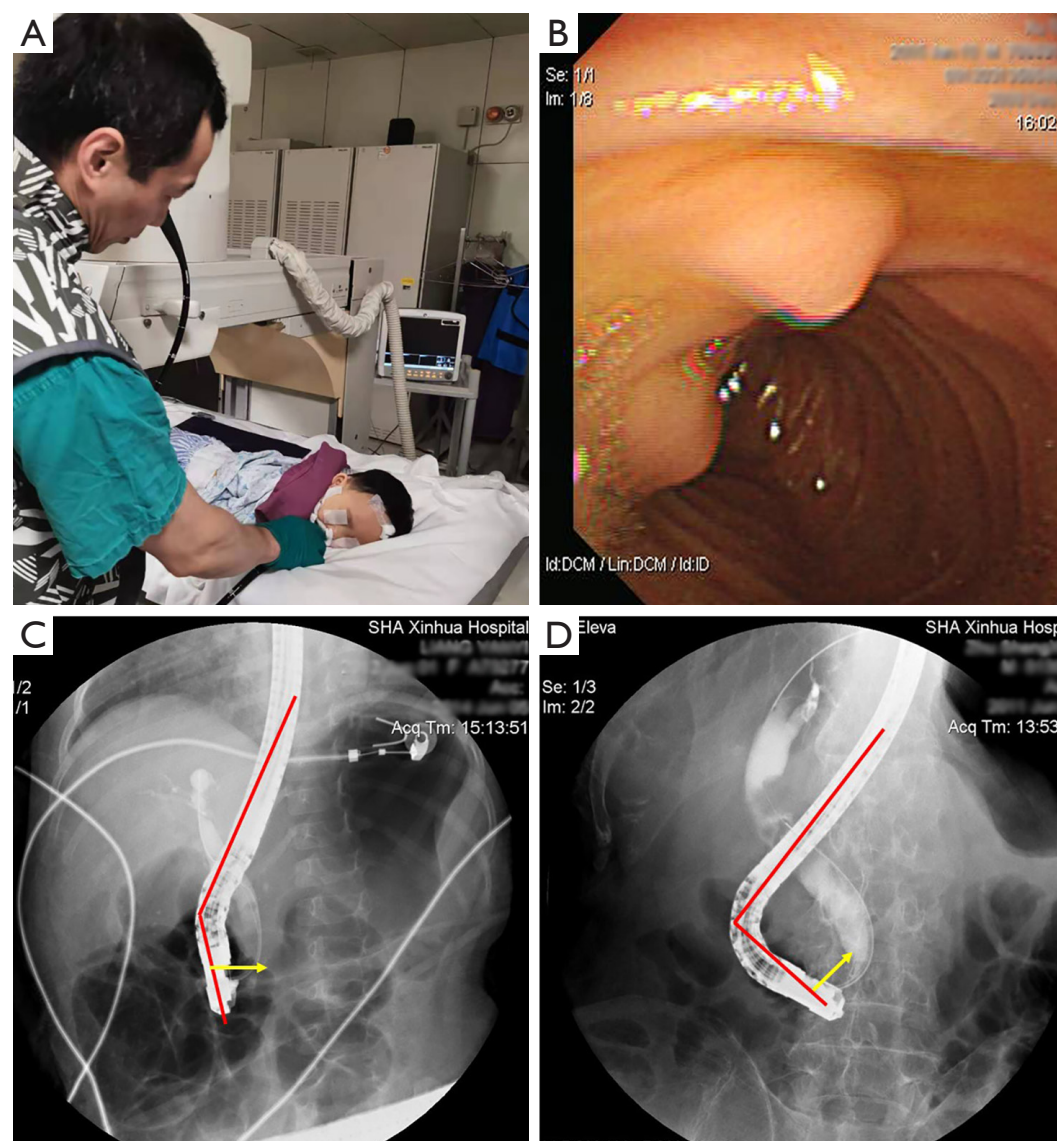

Figure 1 Pictures during ERCP operation. (A) Live performance of ERCP in an infant (8-month-old). (B) Endoscopic view of ERCP in an infant (8-month-old). The endoscope could not go any further. (C) X-ray of ERCP in an infant (8-month-old). (D) X-ray of ERCP in an adult. The yellow arrow indicates the direction of cannulation. This image is published with the consent from the patient's parents. ERCP, endoscopic retrograde cholangiopancreatography.

were between 6 and 12 months of age (mean 10.4 months). The median weight was $6.6(3.3-10.7) \mathrm{kg}$.

The indications for ERCP were biliary infection (five cases), jaundice (three cases), choledochal cysts (three cases), pancreatitis (five cases), and bile duct stricture (one case) (Table 1). Diagnosis after ERCP included choledocholithiasis (six cases), postoperative biliary leak (one case), congenital choledochal cysts (four cases), pancreaticobiliary maljunction (PBM) (eight cases), bile duct stricture (one case), pancreatic duct (PD) stones (four cases), and normal (one case) (Table 2).

Therapeutic ERCP was performed in 15 interventions (88.2\%) and diagnostic ERCP was carried out in two cases (11.8\%). Endoscopic sphincterotomy was performed in 11 procedures (64.7\%). Four patients underwent common bile duct (CBD) stone extraction and three underwent PD stone extraction. Eleven biliary stents and two pancreatic stents were placed during ERCP (Table 2).

\section{Adverse events}

Clinical follow-up was available for all infants (100\%) for at least 72 hours. Complications were recorded in two of the 17 procedures (11.8\%), both of which were diagnosed as mild PEP. One case with pre-existing pancreatitis was excluded. No episodes of moderate or severe PEP happened. All complications were managed conservatively (fasting, antimicrobial therapy, and alleviating protease inhibitor application). There were no complications attributable to mechanical stress on the gastrointestinal tract. Also, no perforations, bleeding, or cardiopulmonary complications occurred (Table 3). 
Table 1 Indications for infant's ERCP

\begin{tabular}{lc}
\hline Indication for ERCP & Number (\%) \\
\hline Biliary infection & $5(29.4)$ \\
Jaundice & $3(17.6)$ \\
Pancreatitis & $5(29.4)$ \\
Choledochal cyst & $3(17.6)$ \\
Bile leak & $1(5.9)$ \\
Total & 17 \\
\hline
\end{tabular}

ERCP, endoscopic retrograde cholangiopancreatography.

Table 2 ERCP diagnoses and interventions

\begin{tabular}{lc}
\hline ERCP diagnoses and interventions & Number $(\%)$ \\
\hline Total & 17 \\
Diagnoses & $1(5.9)$ \\
Normal & $4(23.5)$ \\
Choledochal cyst & $6(35.3)$ \\
CBD stone & $1(5.9)$ \\
CBD stricture & $1(5.9)$ \\
Bile leak & $8(47.1)$ \\
PBM & $6(35.3)$ \\
Pancreatic protease embolus & \\
Interventions & $15(88.2)$ \\
Any & $11(64.7)$ \\
Biliary sphincterotomy & $4(23.5)$ \\
CBD stone extraction & $11(64.7)$ \\
Biliary stent & $3(17.6)$ \\
PD stone extraction & $2(11.8)$ \\
Pancreatic stent & \\
\hline
\end{tabular}

ERCP, endoscopic retrograde cholangiopancreatography; CBD, common bile duct; PBM, pancreaticobiliary maljunction; PD, pancreatic duct.

\section{Discussion}

Over the past decade, some researchers have focused on paediatric ERCP (2-9). Nevertheless, the success rates and frequencies of post-ERCP complications varied significantly due to biased ages, disparate endoscopist experience levels, and indication variations $(4,5)$. For example, the research conducted by Cheng et al. (7) included the largest number
Table 3 Complications of infant's ERCP

\begin{tabular}{lc}
\hline Complications & Number (\%) \\
\hline Pancreatitis & $2(11.8)$ \\
Mild & $2(11.8)$ \\
Moderate & $0(0)$ \\
Severe & $0(0)$ \\
Immediate sphincterotomy bleed & $1(5.9)$ \\
Delayed sphincterotomy bleed & $0(0)$ \\
Esophageal mucosal laceration & $0(0)$ \\
Perforations & $0(0)$ \\
\hline
\end{tabular}

ERCP, endoscopic retrograde cholangiopancreatography.

of cases (329 examinations in 245 children); however, the patient's ages ranged from 5 months to 15 years, with only five cases of choledochal cyst and no cases of biliary atresia (BA). Additionally, a substantial number of patients only underwent diagnostic ERCP, which confuses the identification of success and complication rates. The present study ruled out the biases resulting from age stratification [all patients (age $<1$ year)] as well as operator and equipment distinction, with all procedures being performed with a conventional duodenoscopy by one expert endoscopist. This study aimed to provide effective data on infant ERCP.

The utility of conventional duodenoscopy in infant patients differs from the current management routine. The Chinese 2018 ERCP guidelines suggest that conventional duodenoscopy be used in paediatric patients older than 1 year or heavier than $10 \mathrm{~kg}$, and the evidence grade for this recommendation was A level (15). Several studies have demonstrated that conventional duodenoscopy may not be suitable for infants (14). However, in this study, we achieved a $100 \%$ successful intervention rate, an $11.8 \%$ mild PEP rate, and no severe complications (including uncontrolled bleeding, oesophageal mucosal lacerations, or perforations), which may indicate that conventional duodenoscopy could be safely performed in infant patients $(0.5<$ age $<1$ year). To ensure safety, we suggest that the endoscopist should have at least 5-year and 1,000 cases ERCP experience, and obtain certification for paediatric ERCP. Additionally, all the procedures should rely on the cooperation of the multidisciplinary team, including endoscopists, paediatricians, and paediatric anesthetists.

The diagnoses differed greatly in our study cohort, which mainly included anomalous pancreaticobiliary 
ductal union (47.1\%), CBD stones (35.3\%), PD stones (23.5\%), and congenital choledochal cysts (23.5\%) (Table 2). One obvious difference from other studies is that no BA cases were enrolled in this study. Saito et al. reported that BA accounted for $62.6 \%$ of infant patients (16) while Shteyer et al. reported $48.1 \%$ (17). Our centre prefers to utilize high-end ultrasonography (US) or magnetic resonance cholangiopancreatography (MRCP) as a routine diagnostic method for suspected BA cases, based on the 2017 guidelines for the evaluation of cholestatic jaundice in infants recommended by the North American Society for Pediatric Gastroenterology, Hepatology and Nutrition (NASPGHAN) and European Society for Pediatric Gastroenterology, Hepatology and Nutrition (ESPGHAN) (18). The reported diagnostic accuracy of US for BA varies from $65 \%$ (19) to $98 \%$ (20), and MRCP varies from $71 \%$ (19) to $98 \%$ (21), which is acceptable for clinical diagnosis. Meanwhile, it is worrisome that ERCP could yield false positive findings $(5.35 \%)$ when the contrast does not fill the CBD (22).

Another significant difference between our study and previous research is that a high proportion $(47.1 \%)$ of patients suffered from PBM. PBM is a congenital anomaly defined as a junction of the pancreatic and bile ducts located outside the duodenal wall, usually forming a markedly long common channel, measuring greater than $15 \mathrm{~mm}$ in adults and $5 \mathrm{~mm}$ in children (23). In PBM patients, this anomaly allows regurgitation between the pancreatic and biliary tract, leading to frequent reflux of pancreatic/biliary juice into the bile duct/PD. Therefore, pancreatic enzymes are activated in advance, destroying normal epithelial structures and elastic fibres, and leading to protease emboli, chronic inflammation, and progressive fibrosis. PBM patients are prone to choledocholithiasis, approximately $27.3 \%$ of which is diagnosed as CBD stones (23). In this study, the proportion of PBM with choledocholithiasis was $37.5 \%$ (three of eight cases), and $75 \%$ of PBM patients (six of eight cases) had pancreatic protease emboli. Although few studies have described PBM in infants, this field is expected to attract more research attention and importance because children with PBM will likely develop cancers of the bill duct (3.1\%) and gallbladder (37.4\%) (24). Surgical resection of the extrahepatic bile duct remains the primary management option; however, ERCP plays a crucial role in diagnosis as well as in controlling pancreatitis and jaundice perioperatively.

The paediatric duodenoscope (PJF160) with a working channel of $2 \mathrm{~mm}$ merely allows for the use of specialized equipment hindering therapeutic ERCP. In our cohort, conventional duodenoscopy facilitated the management of stone extraction and stent placement, and consequently, a greater proportion of therapeutic ERCPs $(88.2 \%)$ and a lower diagnostic ERCP rate $(11.8 \%)$ were achieved compared to other studies $(3,4)$. Among 15 therapeutic procedures, endoscopic sphincterotomy was performed in 11 cases, four patients underwent CBD stone extraction, three underwent PD stone extraction, 11 received biliary stents, and two pancreatic stents were placed. The most obvious difference in ERCP between infant and adults lies in the limited space of infant's duodenum, therefore, it was difficult for the scope to hook the third part of the duodenum compared with adults (Figure $1 C, 1 D)$. Consequently, the centre of endoscopic imaging could only point to the underside of the duodenal papilla (Figure 1B), which further complicates cannulation. In our experience, fully bending the head of the papillotome (Dreamtome RX44, Boston-Scientific) could cover the angle and make cannulation and sphincterotome possible.

Clinical follow-up was available for our entire treatment for 3 days. One case with pre-existing acute pancreatitis was excluded from the PEP-related group, and no severe side effects were recorded. The total percentage of complications was $11.8 \%$. Two mild episodes of PEP were observed, which was slightly more frequent than reported rates of PEP in children, ranging from $2.5 \%$ to $9.4 \%$ $(2,7,25)$, and which may be partially attributed to the high percentage of therapeutic procedures performed (88.2\%). Several preprocedural and procedural factors were identified as being related to the development of PEP in the paediatric population. In the univariable analysis, choledochal cysts, recurrent acute pancreatitis and PD cannulation/injection were recognized as risk factors for PEP (Table 4); however, only recurrent acute pancreatitis and PD cannulation/ injection were confirmed by multivariable analysis (Table 5). It is speculated that choledochal cysts and recurrent acute pancreatitis are highly correlated; therefore, they share much of the same explanatory information on PEP, which could explain why choledochal cyst was not ultimately included in the final multiple logistic regression model. As a result, much more attention, for example, urine volume monitoring, ultrasonic examination and so on, should be paid for these two situations.

The rate of PEP significantly increased after PD cannulation or injection. All but two patients in whom mild PEP developed had their PD cannulated, which was consistent with previous studies (13). Interestingly, 
Table 4 Results of the univariate analysis of factors and their influence on development of PEP

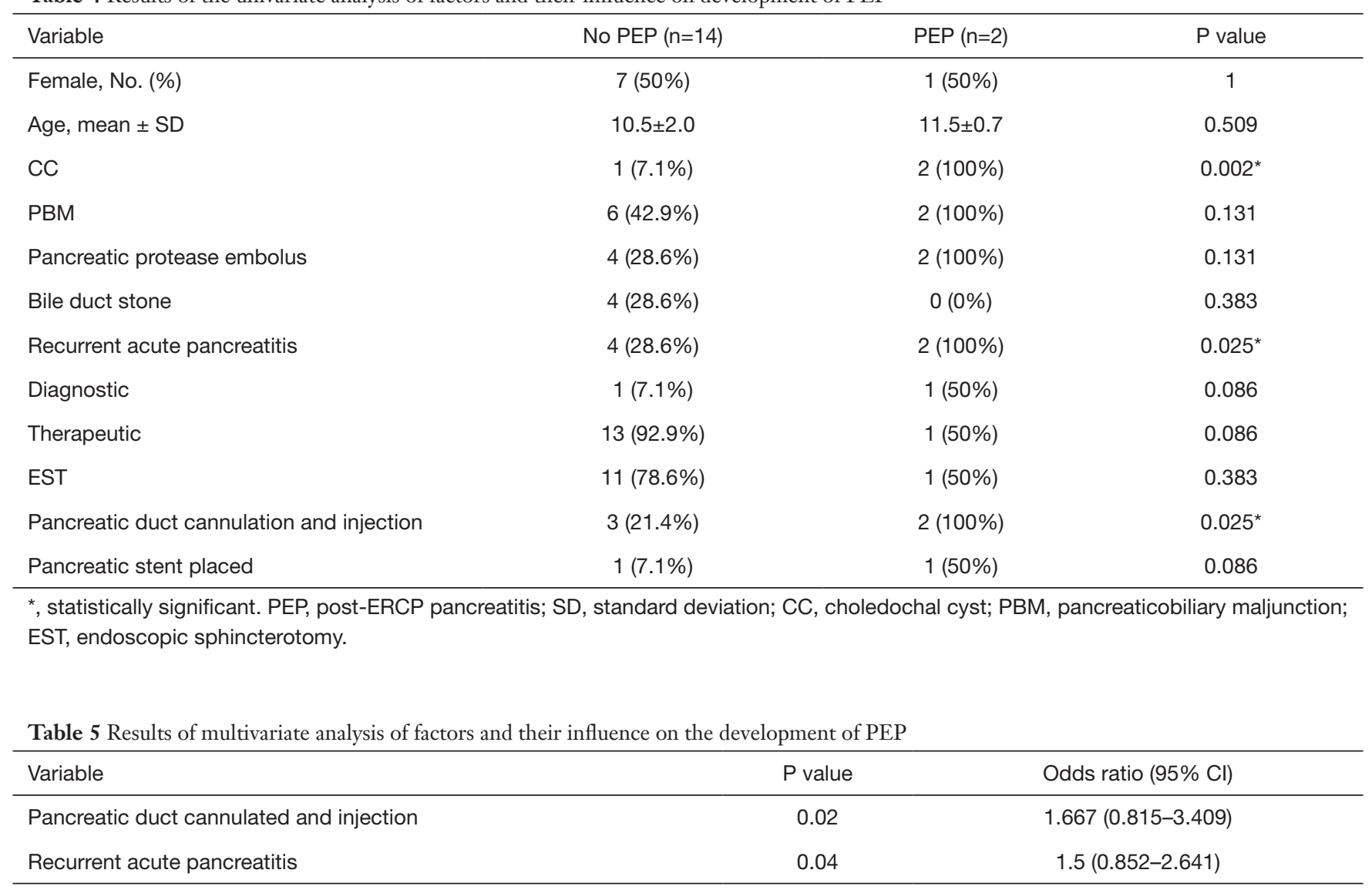

PEP, post-ERCP pancreatitis; Cl, confidence interval.

compared with a large cohort of PEP in children, we observed a non-significant trend towards a protective effect of PD stenting. Rectal nonsteroidal anti-inflammatory drugs (NSAIDs) were not frequently administered in our cohort. We did not find any episodes of perforations, bleeding, ileus, or sepsis; although, delayed complications after discharge could not be formally excluded.

This study has several limitations secondary to the observational study design. Firstly, this was a retrospective study from a single centre with a small number of enrolled infant patients. Also, several procedural variables were not taken into account in order to maintain an efficient study design; for example, difficulty and time of cannulation, details of pre-cut sphincterotomies, as well as accuracy of MRCP and ultrasound. Hence, we will conduct a case-control study to identify the difference between conservational and paediatric endoscopy in paediatric patients older and younger than 1 year.

In summary, our observational study demonstrated that
ERCP could be performed safely and with high success rates by conservational endoscopy in infant patients $(0.5<$ age $<1$ year). A high proportion $(47.1 \%)$ of PBM and therapeutic procedures $(88.2 \%)$ was identified in this cohort. The overall rate of adverse events was acceptable, and no serious adverse events occurred. Recurrent acute pancreatitis and PD cannulation/injection were identified as PEP-related independent risk factors. Even though the beneficial impact of ERCP was identified in the infant cohort, the indication of ERCP and the qualifications of the endoscopists should be seriously considered.

\section{Acknowledgments}

Funding: None.

\section{Footnote}

Reporting Checklist: The authors have completed the 
STROBE reporting checklist. Available at https://dx.doi. org/10.21037/tp-21-406

Data Sharing Statement: Available at https://dx.doi. org/10.21037/tp-21-406

Conflicts of Interest: All authors have completed the ICMJE uniform disclosure form (available at https://dx.doi. org/10.21037/tp-21-406). XW reports that he serves as an unpaid editorial board member of Chinese Fournal of Digestive Endoscopy from 01/2015 to 09/2021. The other authors have no conflicts of interest to declare.

Ethical Statement: The authors are accountable for all aspects of the work in ensuring that questions related to the accuracy or integrity of any part of the work are appropriately investigated and resolved. All procedures performed in this study involving human participants were in accordance with the Declaration of Helsinki (as revised in 2013). The study was approved by ethics committee of the Xinhua Hospital affiliated to Shanghai Jiao Tong University School of Medicine (ID: 201800051). Individual consent for this retrospective analysis was waived.

Open Access Statement: This is an Open Access article distributed in accordance with the Creative Commons Attribution-NonCommercial-NoDerivs 4.0 International License (CC BY-NC-ND 4.0), which permits the noncommercial replication and distribution of the article with the strict proviso that no changes or edits are made and the original work is properly cited (including links to both the formal publication through the relevant DOI and the license). See: https://creativecommons.org/licenses/by-nc-nd/4.0/.

\section{References}

1. Fox VL, Werlin SL, Heyman MB. Endoscopic retrograde cholangiopancreatography in children. Subcommittee on Endoscopy and Procedures of the Patient Care Committee of the North American Society for Pediatric Gastroenterology and Nutrition. J Pediatr Gastroenterol Nutr 2000;30:335-42.

2. Otto AK, Neal MD, Slivka AN, et al. An appraisal of endoscopic retrograde cholangiopancreatography (ERCP) for pancreaticobiliary disease in children: our institutional experience in 231 cases. Surg Endosc 2011;25:2536-40.

3. Paris C, Bejjani J, Beaunoyer M, et al. Endoscopic retrograde cholangiopancreatography is useful and safe in children. J Pediatr Surg 2010;45:938-42.

4. Jang JY, Yoon CH, Kim KM. Endoscopic retrograde cholangiopancreatography in pancreatic and biliary tract disease in Korean children. World J Gastroenterol 2010;16:490-5.

5. Keil R, Snajdauf J, Rygl M, et al. Diagnostic efficacy of ERCP in cholestatic infants and neonates--a retrospective study on a large series. Endoscopy 2010;42:121-6.

6. Iqbal CW, Baron TH, Moir CR, et al. Post-ERCP pancreatitis in pediatric patients. J Pediatr Gastroenterol Nutr 2009;49:430-4.

7. Cheng CL, Fogel EL, Sherman S, et al. Diagnostic and therapeutic endoscopic retrograde cholangiopancreatography in children: a large series report. J Pediatr Gastroenterol Nutr 2005;41:445-53.

8. Varadarajulu S, Wilcox CM, Hawes RH, et al. Technical outcomes and complications of ERCP in children. Gastrointest Endosc 2004;60:367-71.

9. Prasil P, Laberge JM, Barkun A, et al. Endoscopic retrograde cholangiopancreatography in children: A surgeon's perspective. J Pediatr Surg 2001;36:733-5.

10. Aabakken L, Aagenaes I, Sanengen T, et al. Utility of ERCP in neonatal and infant cholestasis. J Laparoendosc Adv Surg Tech A 2009;19:431-6.

11. Guelrud M. Endoscopic retrograde cholangiopancreatography in infants and children. In: Sivak MV, Jr. editor. Gastroenterologic Endoscopy, 2nd ed. Philadelphia: WB Saunders, 2000:1198-220.

12. ASGE Standards of Practice Committee; Anderson MA, Fisher L, et al. Complications of ERCP. Gastrointest Endosc 2012;75:467-73.

13. Troendle DM, Abraham O, Huang R, et al. Factors associated with post-ERCP pancreatitis and the effect of pancreatic duct stenting in a pediatric population. Gastrointest Endosc 2015;81:1408-16.

14. Teng R, Yokohata K, Utsunomiya N, et al. Endoscopic retrograde cholangiopancreatography in infants and children. J Gastroenterol 2000;35:39-42.

15. ERCP Group, Chinese Society of Digestive Endoscopology; Biliopancreatic Group, Chinese Association of Gastroenterologist and Hepatologist; National Clinical Research Center for Digestive Diseases. Chinese guidelines for ERCP (2018). Zhonghua Nei Ke Za Zhi 2018;57:772-801.

16. Saito T, Terui K, Mitsunaga T, et al. Role of pediatric endoscopic retrograde cholangiopancreatography in an era stressing less-invasive imaging modalities. J Pediatr Gastroenterol Nutr 2014;59:204-9. 
17. Shteyer E, Wengrower D, Benuri-Silbiger I, et al. Endoscopic retrograde cholangiopancreatography in neonatal cholestasis. J Pediatr Gastroenterol Nutr 2012;55:142-5.

18. Fawaz R, Baumann U, Ekong U, et al. Guideline for the Evaluation of Cholestatic Jaundice in Infants: Joint Recommendations of the North American Society for Pediatric Gastroenterology, Hepatology, and Nutrition and the European Society for Pediatric Gastroenterology, Hepatology, and Nutrition. J Pediatr Gastroenterol Nutr 2017;64:154-68.

19. Yang JG, Ma DQ, Peng Y, et al. Comparison of different diagnostic methods for differentiating biliary atresia from idiopathic neonatal hepatitis. Clin Imaging 2009;33:439-46.

20. Humphrey TM, Stringer MD. Biliary atresia: US

Cite this article as: Weng $M$, Wang L, Weng $\mathrm{H}$, Gu J, Wang $\mathrm{X}$. Utility of endoscopic retrograde cholangiopancreatography in infant patients with conservational endoscopy. Transl Pediatr 2021;10(10):2506-2513. doi: 10.21037/tp-21-406 diagnosis. Radiology 2007;244:845-51.

21. Han SJ, Kim MJ, Han A, et al. Magnetic resonance cholangiography for the diagnosis of biliary atresia. J Pediatr Surg 2002;37:599-604.

22. Keil R, Snajdauf J, Rygl M, et al. Diagnostic efficacy of ERCP in cholestatic infants and neonates--a retrospective study on a large series. Endoscopy 2010;42:121-6.

23. Kamisawa T, Kaneko K, Itoi T, et al. Pancreaticobiliary maljunction and congenital biliary dilatation. Lancet Gastroenterol Hepatol 2017;2:610-8.

24. Kamisawa T, Kuruma S, Chiba K, et al. Biliary carcinogenesis in pancreaticobiliary maljunction. J Gastroenterol 2017;52:158-63.

25. Issa H, Al-Haddad A, Al-Salem AH. Diagnostic and therapeutic ERCP in the pediatric age group. Pediatr Surg Int 2007;23:111-6. 